matical language to complex numbers. But most physicists have never tried to find an intuitive way of thinking about them; we were always told that it could not be done. Feynman demonstrates that it can, and goes on to introduce another widely used tool in physics, his own Feynman graphs. In a simple and intuitive manner he shows how these diagrams can be "imagined", without understanding what is behind them but getting a good feeling for how they work. Years ago I heard a seminar by Feynman, in which he observed that many physicists used the graphs in much the same way that our ancestors would consult animal entrails for portents of the future. Now it seems that a new understanding has taken over; the graphs are taken as seriously as the arrows, and together they frame the world of present-day physics - the only valid description of nature we have.

Feynman's lectures must have been marvellous and they have been turned into an equally entrancing book, a vivid introduction to QED which is leavened and enlivened by his wit. Anyone with a curiosity about physics today should buy it, not only to get to grips with the deepest meaning of quantum theory but to possess a slice of history.

Pedro Waloschek is a senior scientist at DESY (Deutsches Elektronen Synchrotron), Notkestrasse 85, D-2000 Hamburg 52, FRG.

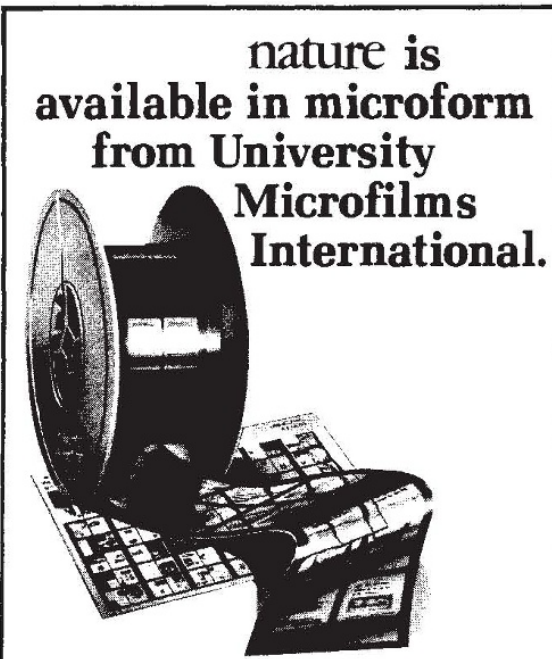

$\square$ Please send information about these titles:

Name

Company/Institution

Address

City

State

Zip

Phonel

Call toll-free 800-521-3044. In Michigan.

Alaska and Hawaii call collect 313-761-4700. Or mail inquiry to: University Microfilms International 300 North Zeeb Road, Ann Arbor, MI 48106.

\title{
Schwinging through relativity
}

\section{Abraham Pais}

Einstein's Legacy: The Unity of Space and Time. By Julian Schwinger. W.H. Freeman: 1986. Pp.250. £15.95.*

THE scientific community appreciates quite well the importance of conveying to a wider audience what science is about. So high are the demands of doing research, however, that few of those active in the field are willing to respond to this need. Doing science and writing about it are actually two distinct activities requiring distinct skills. Nevertheless I like to think that in principle the scientific craftsman is best qualified for popularization. All the many subtleties of any specific scientific subject cannot, in fact should not, be presented to those eager to obtain a first acquaintance with it. Awareness of these subtleties by the popular science writer will, however, temper his style, preventing him from couching his account in those overheated terms and exalted phrases so often used by those who do not really know what is going on.

Julian Schwinger is a pro, particularly well known for his seminal contributions to quantum electrodynamics, for which, with Feynman and Tomonaga, he was awarded the 1965 Nobel Prize in physics. Those as his other writings are marked by consummate mathematical skill. I must confess to considerable astonishment upon receiving his popular account Einstein's Legacy. That, I said to myself, is not the Schwinger I know well. I am happy to report, however, that his book makes for delightful and instructive reading. Technical demands on the reader do not exceed the most elementary algebra.

In the book, parts of Einstein's rich and varied legacy, on quantum theory, on Brownian motion, are touched on only in passing. As the subtitle The Unity of Space and Time indicates, the main thrust is here on relativity theory. The opening chapter "A Conflict Brought to Light" (several of the chapter titles are puns) serves as a prelude to the subject. It describes Michelson and Morley's failure to detect the speed with which the Earth drifts through the ether, that ubiquitous medium, for so long erroneously supposed to be necessary for explaining how light waves travel through space. It is explained why this negative result is of such positive value. Here and elsewhere the author acquaints the reader with the issues by first considering a more familiar analogy, in this case the problem of find-

*Published in the United States as a volume in the Scientific American Library. ing the speed of aircraft moving along with or perpendicular to the jet stream.

The next chapter, "Marking Time", introduces a variety of clocks: pendulums, vibrating atoms, beams circulating in cyclotrons. Soon we are in the midst of the results of relativistic measurements, so different from everyday expectations because of the relativity postulate that the speed of light is unaffected by the motion of the observer. Time intervals dilate, lengths contract - not really, but as seen by an observer at rest who sees, respectively, moving clocks and moving rods. Here, as elsewhere in the book, subtle concepts are elucidated by simple

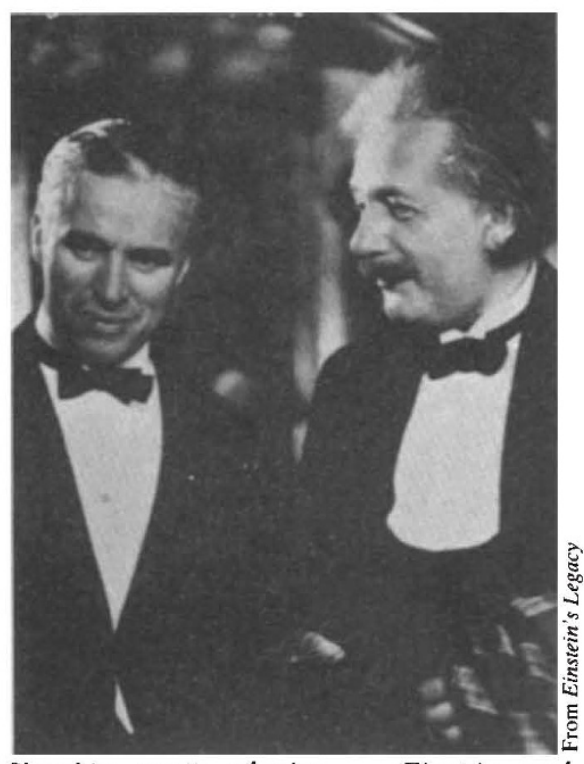

Showbiz meets physics - Einstein and Charles Chaplin in Hollywood, 1931

pictures. Thus the notion of causality is explained by describing the sequence of events at a race track. Cartesian coordinates are introduced by taking the reader on a walk through midtown Manhattan.

A chapter on $E=m c^{2}$ deals with the meaning of that relation in terms of conservation laws and with its applications to (in order of practical importance) chemical, atomic and nuclear reactions, including fission and fusion.

All this, so far, is special relativity which relates experiences of observers in uniform relative motion. As Einstein once said, that theory is child's play compared to general relativity which deals with nonuniform motion. It is particularly gratifying that the subject's intricacies, including glimpses of non-Euclidean and Riemannian geometries, and the theory's early successes, the bending and red shift of light, and the precession of the planet Mercury's perihelion are so well conveyed here in simple language. Altogether, this well printed and pleasingly illustrated book is an ideal gift for the curious non-expert. $\square$

Abraham Pais is a Professor in the Department of Physics, Rockefeller University, 1230 York Avenue, New York, New York 10021, USA. 